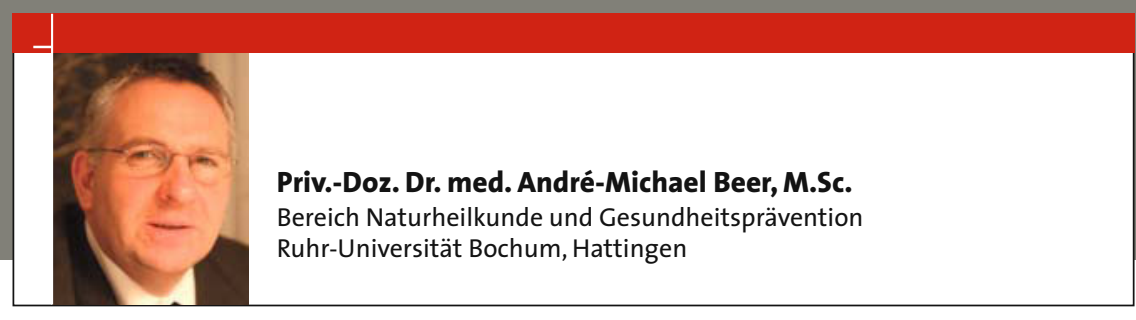

\title{
Jetzt werden die Weichen in der EU gestellt
}

— Viele gute Gründe sprechen für die Anwendung von pflanzlichen Zubereitungen in der ärztlichen Praxis:

- Nach dem Prinzip „Trial and Error“ wurden seit der Antike positive Erfahrungen mit Heilpflanzen kumuliert.

- Moderne Phytopharmaka werden in Deutschland genau wie chemisch-synthetische Arzneimittel über das Arzneimittelgesetz (AMG § 2) und die EU-Richtlinien geregelt.

- Viele Phytopharmaka sind klinisch gut untersucht und als wirksame Arzneimittel durch BfArM, IQWiG und CochraneAnalysen anerkannt.

- Repräsentativen Umfragen zufolge besteht ein großes und nach wie vor steigendes Interesse an pflanzlichen Arzneimitteln. - Phytopharmaka sind meist rezeptfrei - ein Hinweis auf die geringen Risiken, die mit ihrer Einnahme verbunden sind.

- Die Phytotherapie ist mittlerweile fester Bestandteil der universitären Ausbildung (Approbationsordnung 2002).

\section{Das Paradoxon}

Obwohl es eine wissenschaftlich gut dokumentierte Phytotherapie gibt, stehen die Wissenschaft, aber auch Teile der Ärzteschaft, diesem Fachgebiet kritisch gegenüber. Einer der Gründe ist, dass Phytopharmaka Vielstoffgemische sind, die nach herkömmlicher Meinung der Pharmakologie eine rationale Beurteilung nicht zulassen. Dies ist falsch, wenn man die Besonderheiten der Phytotherapie angemessen berücksichtigt: Der Extrakt ist der Wirkstoff; verschiedene Extrakte aus der gleichen Heilpflanze können unterschiedliche Wirkprofile aufweisen; pflanzliche Extrakte haben i.d.R. ein breites Wirkprofil.

Die Phytotherapie hat ihre eigenen Gesetzmäßigkeiten, die es zu beachten gilt. Inzwischen konnten viele Wirkmechanismen pflanzlicher Extrakte entschlüsselt und deren Wirksamkeit in kontrollierten klinischen Studien belegt werden. Die Phytotherapie leidet v. a. aber unter ihrem Zwitterdasein: Phytopharmaka werden zwar nach den gleichen Kriterien wie chemisch-synthetische Arzneimittel beurteilt, regulatorisch gehören sie aber zu den besonderen Therapierichtungen. Ihre Vertreter suchen ihre Position im Rahmen des EU-Harmonisie- rungsprozesses eher im Bereich der CAM (Complementary and Alternative Medicine), die auch Verfahren wie Homöopathie, anthroposophische Medizin oder Akupunktur umfasst. Hier besteht das Problem, dass nach EU-Einschätzung „die Wissensgrundlage für CAM-Verfahren unsicher bleibt und Gegenstand von Spekulationen anstelle von gesichertem Wissen ist" (Kommentar der Initiative für komplementär- und alternative Methoden in der EU). Zudem wird der Begriff „Komplementärmedizin“ nach Definition der National Library of Medicine (USA) für „alle jene Praktiken der gesundheitlichen Versorgung verwendet, die nicht Bestandteil der konventionellen Medizin sind“. Und der ebenfalls oft genannte Begriff „unkonventionelle Medizin“ umfasst nach Interpretation der Europäischen Kommission „einen sehr heterogenen Sektor mit dem gemeinsamen Merkmal: Ausschluss aus der konventionellen, etablierten wissenschaftlichen Medizin und spärliche Repräsentanz in der universitären Lehre und Forschung“.

Unter Lobbygesichtspunkten scheint es also angebracht, sich im Rahmen der naturheilkundlichen EU-Initiativen (Camdoc) unter den komplementärmedizinischen Maßnahmen einzuordnen, um überhaupt Beachtung zu finden. Der Preis dafür ist aber, dass die Phytotherapie, wie alle im CAM zusammengefassten Verfahren, durch diese Definition der EU einen unwissenschaftlichen Anstrich erhält.

\section{Wer soll die Phytotherapie vertreten?}

Ein wesentlicher Schritt wäre sicherlich der Aufbau einer eigenen Vertretung wie z.B. einer europäischen Ärztegesellschaft für Phytotherapie. Dabei muss v.a. den Anwendern Folgendes bewusst sein: Wird die Phytotherapie in Brüssel zum jetzigen Zeitpunkt nicht ausreichend vertreten, wird man sich in einer Position wiederfinden, in der man mühsam versuchen muss, bereits getroffene Entscheidungen zu revidieren oder Schadensbegrenzung zu betreiben. Damit verschenkt man den eingangs geschilderten Bonus einer gut dokumentierten und wissenschaftlich begründeten Heilmethode, die kostengünstig, nebenwirkungsarm und vom Patienten erwünscht ist. 